Angelika Storrer

\title{
Wie notwendig sind obligatorische Valenzstellen? - Faktoren der Weglaßbarkeit von Valenzstellen im Text
}

\section{Einleitung}

Die von Tesnière vorgestellte Dependenzgrammatik ist vornehmlich eine Analysegrammatik, die sich auf die Beschreibung von Strukturen bereits vorliegender Sätze konzentriert. In diesem Rahmen konnte die Frage unbeantwortet bleiben, ob und unter welchen Bedingungen die im Stellenplan eines Verbs vorgesehenen Valenzstellen zu realisieren sind. Tesnière war jedoch mitnichten der Ansicht, daß ein Verb immer mit allen seinen Aktanten vorkommen müsse; vielmehr baut er seine Valenzmetapher weiter aus, indem er zwischen gesättigten und ungesättigten Valenzstellen unterscheidet:

„Notons d'ailleurs qu'il n'est jamais nécessaire que les valences d'un verbe soient toutes pourvues de leur actant et que le verbe soit, pour ainsi dire, saturé. Certaines valences peuvent rester inemployées ou libres." 1

Die Frage, welche Faktoren die Sättigung der Valenzstellen eines Verbs determinieren, ließ er allerdings offen.

Die lexikographische und die grammatikographische Anwendung der Valenzidee, speziell für den Fremdsprachenunterricht, ließ es jedoch wünschenswert erscheinen, Valenzstellen von Verben zu unterteilen in solche, die in jeder Äußerung zusammen mit dem Verb realisiert sein müssen (z.B. das Akkusativkomplement bei ehelichen), und solche, die unter bestimmten Bedingungen auch weggelassen werden können (z.B. das Akkusativkomplement bei heiraten). Ähnliches gilt für valenzorientierte Verbbeschreibungen, die für die maschinelle Sprachverarbeitung bestimmt sind. Die in der Nachfolge Tesnières präferierte Lösung des Problems war die Differenzierung zwischen obligatorischen und fakultativen Valenzstellen - eine Unterscheidung, die jedoch in der Geschichte der anwendungsorientierten Valenzforschung bis heute ebenso umstritten ist wie die Abgrenzung zwischen valenzgebundenen Komplementen und nicht valenzgebundenen Supplementen.

Im folgenden Abschnitt werden die einschlägigen Probleme angesprochen, die mit der klassischen Herangehensweise an die Frage der Valenzstellenrealisierung, der obligatorisch/fakultativ-Klassifikation, verbunden sind. In Abschnitt 3 wird als Alternative dazu ein Modell zur Diskussion gestellt, das diejenigen Parameter in ihrem Zusammenspiel beschreibt, die die Wahl der in einer Äußerung zu realisierenden Verb-Rollen-Konstellation entscheidend beeinflussen. Dem Modell liegt die Annahme zugrunde, daß die Entscheidung, welche und wieviele Valenzstellen in einer Äußerung zu realisieren sind, in erster Linie durch kommunikativ-pragmatische Faktoren gesteuert wird; syntaktische und semantische Faktoren kommen erst in zweiter Linie zum Tragen. Vor dem Hintergrund die-

1 Tesnière (1959: 238); vgl. auch die Stemmata für die Sätze Alfred donne aux pauvres. und Alfred donne la main. in Tesnière (1959: 239). 
ses Modells wird in Abschnitt 4 erläutert, warum die Weglaßbarkeit von Valenzstellen mit Hilfe des syntaktisch-strukturell motivierten Eliminierungstests (auch Reduktionstest oder Weglaßprobe genannt) nicht zuverlässig $\mathrm{zu}$ ermitteln ist und weshalb die Unterscheidung zwischen definiter und indefiniter Auslassung von Valenzstellen nicht auf lexikalischer Ebene angesiedelt werden kann. ${ }^{2}$

\section{Die Klassifikation in obligatorische und fakultative Valenzstellen}

Die klassische Herangehensweise an die Frage nach der Valenzrealisierung, im weiteren obligatorisch-fakultativ-Klassifikation (o/f-Klassifikation) genannt, besteht darin, die Valenzstellen eines Verbs in obligatorische und fakultative zu unterteilen. Sie findet sich in den meisten Valenzwörterbüchern, wobei üblicherweise - in Analogie zur Notation bei Phrasenstrukturregeln - die fakultativen Elemente geklammert erscheinen. ${ }^{3}$ Mit der o/f-Klassifikation ist eine Regel zur Realisierung von Valenzstellen verbunden, die sich folgendermaßen formulieren läßt:

1. In einem grammatischen Satz mit einem Verb V müssen alle obligatorischen Valenzstellen von $\mathrm{V}$ realisiert sein.

2. Fakultative Valenzstellen können unter bestimmten Bedingungen fehlen, sind dann jedoch aus dem Sprach- oder Situationskontext entnehmbar.

3. Nicht valenzgebundene Supplemente sind frei hinzufüg- und weglaßbar.

Gegen diese Regel lassen sich verschiedene Einwände vorbringen: ${ }^{4}$

1. Die Realisierung bestimmter Supplementklassen (z.B. Instrumentale, Direktionale) unterliegt verbsubklassenspezifischen Beschränkungen. Die freie Hinzufügbarkeit von Supplementen muß entsprechend eingeschränkt werden.

2. Auch die beliebige Weglaßbarkeit von Supplementen besteht nur bei isolierten Sätzen, wie sie üblicherweise im linguistischen Versuchslabor produziert werden. In Sätzen, die Teil eines größeren Redezusammenhangs sind, können Supplemente eine tragende Rolle für die Kohärenz von Texten spielen und deshalb unter kommunikativen Gesichtspunkten nicht weggelassen werden.

3. Die Auffassung, fehlende fakultative Ergänzungen seien immer aus dem sprachlichen oder dem Situationskontext erschließbar, muß durch die Unterscheidung zwischen definiter und indefiniter Auslassung von Valenzstellen relativiert werden: ${ }^{5}$

${ }^{2}$ Für anregende Diskussionen und wertvolle Hinweise bedanke ich mich bei Joachim Jacobs, Ulrich Klein und Kerstin Blume vom Projekt Syntaktische Valenz im Lexikon des SFB 282 an der BUGH Wuppertal.

${ }^{3}$ Vgl. z.B. die Satzbauplanangaben in HS, BW und ViF.

4 Für eine ausführliche Diskussion vgl. Storrer (1992: 95-105; 238-256).

5 Vgl. Fillmore (1971), Sæbø (1984). 
(1) Das Eichhörnchen konnte die verstecken Haselnüsse nicht mehr finden. Den ganzen Tag suchte es vergebens.

(2) Das Eichhörnchen hat sich von seiner Krankheit erholt. Jetzt ißt es wieder.

In (1) ist die Belegung des Präpositionalkomplements von suchen tatsächlich aus dem Kontext rekonstruierbar; es handelt sich somit um eine definite Auslassung der entsprechenden Valenzstelle.

Anders beim fehlenden Akkusativkomplement von essen in (2): Hier ist die Belegung der Valenzstelle für den aktuellen Redezusammenhang irrelevant und kann deshalb sowohl dem Sprecher als auch dem Hörer unbekannt sein und auch bleiben. Es handelt sich um eine indefinite Auslassung der entsprechenden Valenzstelle.

4. Empirische Untersuchungen, die an als akzeptabel und korrekt befundenen Korpussätzen durchgeführt wurden, zeigen, daß auch obligatorische Valenzstellen unter bestimmten kontextuellen und kommunikativen Voraussetzungen fehlen können, bzw. unter kommunikativen Gesichtspunkten sogar fehlen müssen.

Insgesamt zeigt sich, daß die mit der o/f-Klassifikation verbundene Regel zur Realisierung von Valenzstellen die sprachlichen Daten nicht adäquat erfaßt. Ein extensiver Vergleich von Valenzangaben in den Wörterbüchern HS, ViF und BW ergab außerdem, daß $20 \%$ der in allen drei Wörterbüchern aufgeführten Verben widersprüchlich hinsichtlich der vorgenommenen o/f-Klassifikation sind. ${ }^{6}$ Offensichtlich können also mit dem zur Klassifikation herangezogenen Verfahren, dem Eliminierungstest, keine konsistenten Klassifikationsergebnisse erzielt werden, so da $\beta$ dieselbe Valenzstelle eines Verbs in verschiedenen Wörterbüchern einmal als obligatorisch, einmal als fakultativ eingestuft wird. All diese Mängel machen deutlich, daß die o/f-Klassifikation die Bedingungen der Valenzrealisierung nicht adäquat beschreibt; dies umso mehr, wenn neben grammatischer Korrektheit auch kommunikative Adäquatheit erfaßt werden soll.

\section{Das Modell der Situationsvalenz}

Als Grund für die Unzulänglichkeit der o/f-Klassifikation wurde schon relativ früh herausgestellt, daß bei der Frage nach der Valenzrealisierung kommunikativ-pragmatische, semantische und syntaktische Faktoren in komplexer Weise zusammenspielen, denen eine dichotomischen Unterscheidung nicht gerecht werden kann. ${ }^{7}$ Das Modell der Situationsvalenz versucht, verschiedene Erkenntnisse über den Zusammenhang von Valenz und Text bzw. Valenz und Kommunikation in ein einheitliches Modell zu integrieren. Es beschreibt diejenigen Parameter in ihrem Zusammenspiel, die die Wahl der in einer Äußerung zu realisierenden Verb-Rollen-Konstellation entscheidend beeinflussen. Die Modellierung ist von der Grundannahme geleitet, daß die Auswahl der in einer Äußerung zu realisierenden

\footnotetext{
6 Detaillierte Ergebnisse finden sich in Storrer (1992), Kapitel 5.

7 Vgl. Heringer (1984), von Polenz (1985), Helbig (1988).
} 
Valenzstellen in erster Linie durch kommunikativ-pragmatische Faktoren gesteuert wird, erst dann kommen syntaktische und semantische Merkmale zum Tragen.

Im folgenden werden die Grundideen des Modells soweit skizziert, wie sie für das weitere Verständnis benötigt werden. ${ }^{8}$ Sprachtheoretisch schließt das Modell an die Kommunikantensemantik (Mudersbach 1984) an; die im Modell skizzierte Repräsentation von Wissen über Situationen und Situationstypen orientiert sich an Frame-Konzepten aus der sog. künstlichen Intelligenz.

Ausgangspunkt des Modells ist eine Äußerungssituation A-Sit, in der ein Sprecher S mit einem Hörer $\mathbf{H}$ bzw. einem Kollektiv von Hörern über ein bestimmtes Redethema kommuniziert. Der Sprecher will dabei dem Hörer zu einer bestimmten Situation (im weiteren Rekurssituation R-Sit genannt) etwas Neues und Relevantes mitteilen und konzipiert dazu eine entsprechende Äußerung U. Zu den valenzrelevanten Parametern gehören nun verschiedene Wissensbestände, die dem Sprecher aufgrund seiner Zugehörigkeit zu sprachlichen und kulturellen Gemeinschaften einerseits und seiner individuellen Biographie andererseits zur Verfügung stehen. Andere valenzrelevante Parameter beziehen sich auf die Äußerungssituation selbst: Hierzu zählen Zeitpunkt und Ort der Äußerung, das aktuelle Redethema, die Interessenslage des Sprechers und seine Hypothesen über die Interessenslage des Hörers.

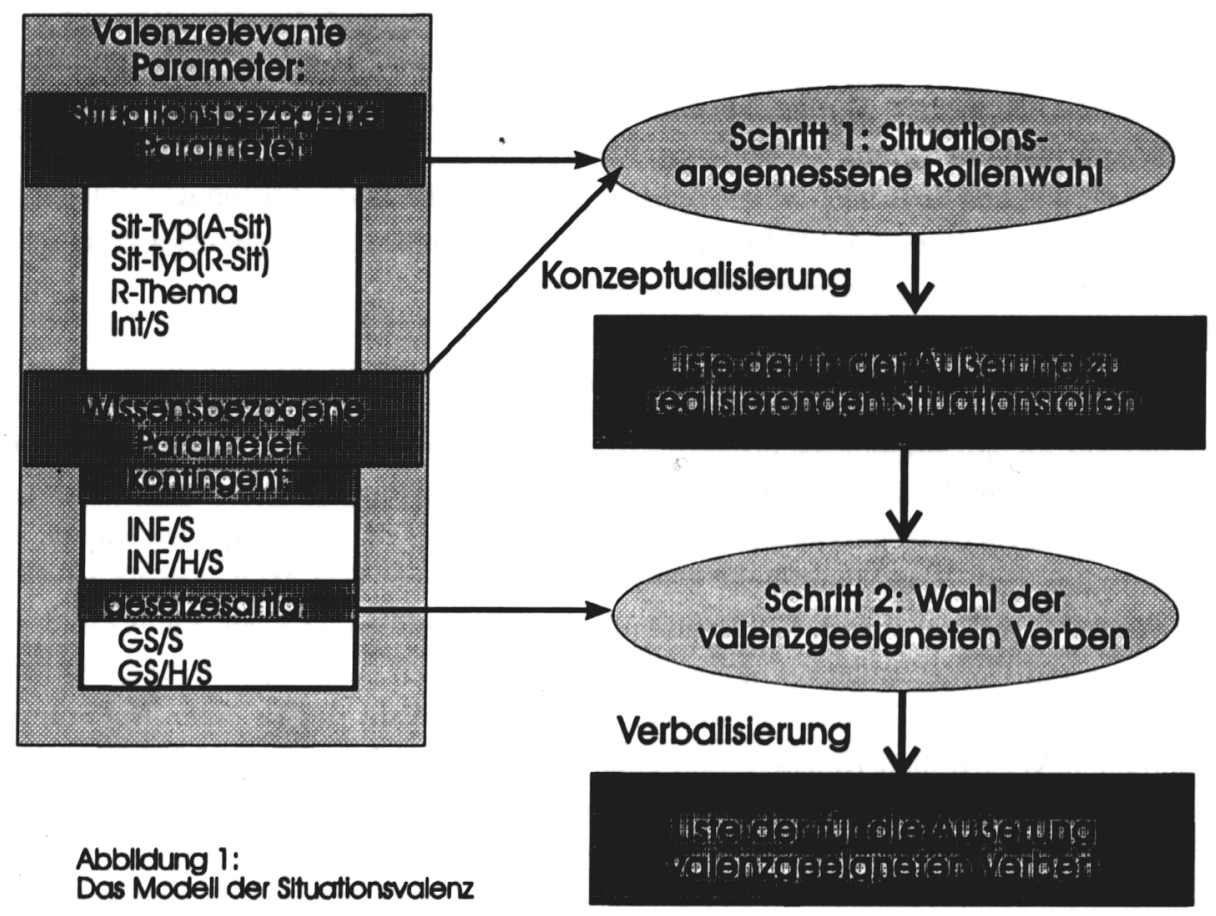

\footnotetext{
${ }^{8}$ Eine ausführliche Darstellung findet sich in Storrer (1992), Kapitel 8.
} 
In Abbildung 1 sind die Modellkomponenten und deren Zusammenspiel graphisch dargestellt. Das Modell umfaßt eine statische Komponente, in der alle valenzrelevanten $\mathrm{Pa}$ rameter für die jeweilige Äußerungssituation instantiiert werden, und eine dynamische Komponente, die in zwei Schritten über diesen Parameterwerten operiert:

- Im ersten Schritt, der kommunikativ angemessenen Rollenwahl, wird entschieden, welche Situationsrollen für eine kommunikativ angemessenen Äußerung zu wählen sind; der Begriff der kommunikativen Angemessenheit orientiert sich dabei an den in Grice (1975) postulierten Konversationsmaximen.

- Im zweiten Schritt, der Wahl der valenzgeeigneten Verben, wird dann entschieden, mit welchen Verben die in Schritt 1 gewählte Rollenkonstellation angemessen verbalisiert werden kann.

Der erste Schritt bewegt sich auf der übereinzelsprachlich konzeptuellen Ebene, während für den zweiten Schritt die Schnittstelle zwischen konzeptuell verstandenen Situationsrollen und den Valenzstellen entsprechender einzelsprachspezifischer Verben von entscheidender Bedeutung ist.

\subsection{Kommunikativ angemessene Rollenwahl}

Ein Sprecher verfügt normalerweise über die Fähigkeit, aufgrund seines Erfahrungswissens eine Situation unter einen generischen Situationstyp zu subsumieren, zu welchem ihm eine Reihe prototypischer Situationsrollen bekannt ist. Ein zur Illustration oft herangezogener Situationstyp ist die sog. Kaufszene mit den prototypischen Rollen des Käufers, des Verkäufers, des Geldwerts und der Ware. Ausgangspunkt von Schritt 1 ist die Menge genau der Situationsrollen, die dem Sprecher zum Situationstyp der Rekurssituation (d.h. der Situation, über die kommuniziert wird) bekannt sind. In Schritt 1 durchläuft jede dieser Situationsrollen mehrere Filter, wobei jeweils der Wert eines bestimmten valenzrelevanten Parameters darüber entscheidet, ob die Rolle in der geplanten Äußerung zu realisieren ist oder nicht. Haben alle Situationsrollen die Filter durchlaufen, steht das Inventar der in der Äußerung zu realisierenden Situationsrollen fest - dieses Inventar ist dann wiederum Eingabe für den zweiten, einzelsprachspezifischen Schritt des Modells. Im einzelnen werden die folgenden Parameterwerte abgeprüft:

1. Der Sprecherwissensfilter prüft, ob die Belegung der Rolle dem Sprecher überhaupt bekannt ist. Ist dies nicht der Fall, kann die Rolle höchstens erfragt oder als Indefinitpronomen (z.B. etwas, jemand) verbalisiert werden; ansonsten wird die jeweilige Rolle herausgefiltert.

2. Der Hörerwissensfilter prüft, ob die Belegung der Rolle dem Hörer bereits bekannt ist. ${ }^{9}$ Ist dies der Fall, so wird er sie im allgemeinen nicht verbalisieren, da er dem Hörer

9 Dabei wird, im Sinne der Modellierung von Sprecher- und Hörerwissen in der Kommunikantensemantik, nicht der Informationsstand des Hörers selbst, sondern der hypothetische Informationsstand des Hörers aus Sprechersicht geprüft, vgl. Mudersbach (1984). Ebenso wird im Gesetzeswissensfilter der 
ja nicht Bekanntes, sondern etwas Neues mitteilen möchte. Er kann die entsprechende Rolle höchstens in thematischer Funktion verwenden, d.h., um den Anschluß an vorhergehende Text- und Redeteile herzustellen. ${ }^{10}$ Ist der thematische Anschluß bereits gewährleistet, wird die betreffende Situationsrolle herausgefiltert.

3. Der Relevanzfilter prüft, ob der Sprecher die Belegung der Rolle in bezug auf das aktuelle Redethema als relevant befindet; ist dies nicht der Fall, wird die betreffende Rolle herausgefiltert.

4. Der Situationsfilter greift in dem Spezialfall, daß Äußerungs- und Rekurssituation identisch sind, d.h., daß über die Gegebenheiten der Äußerungssituation kommuniziert wird. In diesem Fall kann die Belegung der in Frage stehenden Rolle aus der Äußerungssituation direkt entnommen werden; sie muß daher nicht mehr verbalisiert werden. Rollen, deren Belegung sich direkt aus der Äußerungssituation erschließen lassen, werden herausgefiltert.

5. Der Gesetzeswissensfilter operiert über dem Gesetzeswissen des Hörers und zwar speziell über dessen Wissen von standardmäßigen Situationsabläufen und standardmäßigen Rollenbelegungen. Eine Situationsrolle, deren Belegung sich aus dem Wissen über standardmäßige Rollenbelegungen ergibt, wird herausgefiltert.

6. Der Interessensfilter prüft, ob es der aktuellen Interessenslage des Sprechers widerspricht, dem Hörer die Belegung der Rolle bekannt werden zu lassen. Die Interessenslage wird bestimmt durch den spezifischen Zweck, den der Sprecher mit seiner Äußerung, verstanden als Teil einer komplexen Sprachhandlung, verfolgt, sowie durch die soziale Rolle, die er in der Äußerungssituation innehat. Läuft es dem Interesse des Sprechers zuwider, den Hörer über die Belegung einer Situationsrolle in Kenntnis zu setzen, so wird diese durch den Interessensfilter herausgefiltert.

Haben alle Situationsrollen, die dem Sprecher zum Situationstyp der Rekurssituation bekannt sind, jeweils alle sechs Filter durchlaufen, steht das Inventar der in der Äußerung zu realisierenden Rollen fest.

\subsection{Wahl eines valenzgeeigneten Verbs}

Im zweiten Schritt der dynamischen Modellkomponente wird entschieden, wie sich das im ersten Schritt festgelegte Inventar an konzeptuellen Situationsrollen in einer Einzelsprache angemessen verbalisieren läßt.

hypothetischen Gesetzesbereich des Hörers aus Sprechersicht geprüft und nicht der Gesetzesbereich des Hörers selbst, der ja dem Sprecher während der Konzeptualisierungsphase nicht direkt zugänglich ist.

10 Die Termini thematisch und rhematisch werden hier im Sinne der informationszogenen Thema-RhemaTheorie (Mudersbach 1981, vgl. Gerzemysch 1987) verwendet. 
Folgende Bereiche sprachlichen Wissens werden zur Beantwortung dieser Frage herangezogen:

- Wissen darüber, welche Verben einen bestimmten Situationstyp beschreiben können. Diejenigen Verben einer Einzelsprache, die aufgrund ihrer semantischen Eigenschaften prinzipiell für die Bezeichnung eines bestimmten Situationstyps in Betracht kommen, sind bezeichnungsgeeignet in bezug auf den jeweiligen Situationstyp.

- Wissen darüber, mit welchen Valenzstellen der Verben welche Situationsrollen des jeweiligen Situationstyps verbalisiert werden können, modelliert als funktionale Abbildung der Menge der verbspezifischen Valenzstellen in die Menge der Situationsrollen.

- Kenntnis der formalen und inhaltlichen Merkmale dieser einzelverbspezifischen Valenzstellen, d.h. Angabe von syntaktischer Kategorie und syntaktischer Funktion, sowie semantische Belegungsregeln.

Die bezeichnungsgeeigneten Verben für einen Situationstyp können sich in mancherlei Hinsicht unterscheiden. Für die Wahl der valenzgeeigeneten Verben sind die folgenden Oppositionen relevant:

1. Oppositionen im Hinblick auf die Art und Anzahl der Valenzstellen, die mit den einzelnen bezeichnungsgeeigneten Verben angeschlossen werden können. So ist beispielsweise im Stellenplan des Verbs anordnen - im Gegensatz zu befehlen - keine Valenzstelle für die Rolle des Befehlsempfängers angelegt.

2. Oppositionen im Hinblick auf die Perspektive, die mit den verschiedenen Verben auf den jeweiligen Situationstyp geworfen wird. Im Deutschen werden mit manchen Verben bestimmte Situationsrollen fest perspektiviert, d.h., mit der Wahl dieses Verbs muß die betreffende Valenzstelle auf jeden Fall verbalisiert werden; diese Verben werden als perspektivierungsfixiert in bezug auf den jeweiligen Situationstyp bezeichnet. So ist beispielsweise das Akkusativkomplement des Verbs ehelichen - im Gegensatz zum Verb heiraten - perspektivierungsfixiert in bezug auf die Rolle des Ehepartners. ${ }^{11}$

Für die Wahl der valenzgeeigneten Verben werden im zweiten Schritt der dynamischen Modellkomponente alle Verben, die in bezug auf die in Frage stehende Rekurssituation bezeichnungsgeeignet sind, auf die folgenden beiden Bedingungen hin abgeprüft:

1. Alle im ersten Schritt gewählten Situationsrollen sind als verbspezifische Valenzstellen verbalisierbar.

2. Keine der im ersten Schritt nicht gewählten Situationsrollen ist einer perpektivierungsfixierten Valenzstelle zugeordnet.

11 Die meisten der perspektivierungsfixierten Valenzstellen sind zwar in herkömmlichen Valenzwörterbüchern als obligatorisch markiert, jedoch sind bei weitem nicht alle obligatorisch markierten Valenzstellen auch perspektivierungsfixiert. $\mathrm{Zu}$ den zentralen Unterschieden zwischen den Termini perspektivierungsfixiert und obligatorisch vgl. Storrer (1992: 314ff.). 
Alle bezeichnungsgeeigneten Verben, die beiden Bedingungen genügen, sind valenzgeeignet relativ zu der im ersten Schritt getroffene Wahl an Situationsrollen. Die erste Bedingung muß erfüllt sein, da der Sprecher ansonsten Informationsteile weglassen müßte, die er auf der konzeptuellen Ebene für seine Äußerung bereits gewählt hatte. Ist die zweite Bedingung bei einem bezeichnungsgeeigneten Verb nicht erfüllt, dann ist der Sprecher mit der Wahl dieses Verbs gezwungen, Informationsteile anzuschließen, die er für seine Äußerung - aus gutem Grund - nicht gewählt hatte. Beide Effekte sind unter kommunikativen Gesichtspunkten nicht wünschenswert; auch für die Übersetzung einer in der Ausgangsprache vorgegebenen Konstellation von Situationsrollen in eine Zielsprache müssen beide Bedingungen erfüllt sein.

Das Modell ist so angelegt, daß sich durchaus mehrere bezeichnungsgeeignete Verben als valenzgeeignet erweisen können: Wurden beispielsweise im ersten Schritt die Situationsrollen des Lügners und des Belogenen als Beteiligte eines Lügen-Szenarios ausgewählt, so werden sich für das Deutsche die Verben belügen, anlügen, anschwindeln gleichermaßen als valenzgeeignet anbieten. Welches dieser Verben dann letzlich für die Äußerung zu wählen ist, wird von Faktoren gesteuert, die unabhängig von der Valenz der Verben sind und außerhalb des Gegenstandsbereichs der Valenzforschung liegen.

\section{Konsequenzen für die obligatorisch-fakultativ-Dichotomie}

Die Entscheidung darüber, welche konzeptuellen Rollen für eine kommunikativ angemessene Äußerung zu wählen sind, ist - wie in Schritt 1 des Modells gezeigt wurde größtenteils abhängig von spezifischen Parametern der Äußerungs- und der Rekurssituation: Dem Informationsstand des Sprechers und des Hörers, dem aktuellen Redethema, den räumlichen und zeitlichen Koordinaten der Äußerungssituation sowie der momentanen Interessenlage des Sprechers. Aus dieser Beobachtung ergeben sich einige interessante Konsequenzen für die lexikographische und grammatikographische Beschreibung der entsprechenden Regularitäten, die im folgenden weiter ausgeführt werden sollen.

4.1 Konsequenzen für die Unterscheidung zwischen definiter und indefiniter Fakultativität

Die in Abschnitt 3 erwähnte Unterscheidung zwischen definit-fakultativer und indefinitfakultativer Auslassung von Valenzstellen wird im Modell der Situationsvalenz folgendermaßen erfaßt:

- Indefinite Auslassung liegt vor bei den Rollen, die durch den Relevanzfilter herausgefiltert worden sind, weil sie für das aktuelle Redethema nicht relevant waren.

- Definite Auslassung liegt vor bei den Rollen, die durch den Hörerwissensfilter, den Situationsfilter oder den Gesetzeswissensfilter herausgefiltert wurden; die Belegung dieser Rollen kann, zumindest aus der Sicht des Sprechers, jeweils aus dem Sprach- oder Situationskontext entnommen werden. 
Das Modell der Situationsvalenz zeigt jedoch, daß ohne Rückgriff auf Parameter der Rekurs- und Äußerungssituation nicht entschieden werden kann, aus welchem Grund eine konzeptuelle Rolle für eine bestimmte Äußerung gewählt oder nicht gewählt worden ist. Daraus ergibt sich, daß der von Fillmore (1971) und Sæbø (1984) eingebrachte Vorschlag nicht tragfähig ist, fakultative Valenzstellen im Lexikon als entweder definit-fakultativ oder als indefinit-fakultativ zu subklassifizieren. Ein solches Vorgehen liefe darauf hinaus, auf der Ebene des Lexikons festzuschreiben, aus welchem Grund eine bestimmte Rolle in einer Äußerung, d.h. auf der Ebene des Textes, fehlt. Eine derartige Festschreibung ist nicht möglich; vielmehr kann ein und dieselbe Valenzstelle in verschiedenen Äußerungen entweder definit oder indefinit ausgelassen werden, wie die folgenden Beispiele zeigen: ${ }^{12}$

(3) Fritz geht den Hund füttern. Er bringt das Fleisch.

$(\rightarrow$ definit: dem Hund)

(4) Am Heiligabend brachte der Weihnachtsmann Geschenke.

$(\rightarrow$ indefinit: irgendwem)

(5) Peter zündet sich die Zigarette an und raucht.

$(\rightarrow$ definit: die Zigarette)

(6) Peter sitzt vor dem Radio. Er raucht.

$(\rightarrow$ indefinit: irgendwas)

Statt definiter und indefiniter Fakultativität auf der Ebene des Lexikons lassen sich deshalb lediglich verschiedene Typen von Auslassungen auf der Ebene des Textes unterscheiden. Das Modell der Situationsvalenz erlaubt dabei sogar eine feinere Ausdifferenzierung der definit-indefinit-Dichtotomie, da die Faktoren, die zur definiten Auslassung einer Rolle im Textsatz führen können, durch die verschiedenen Filter feiner differenziert werden.

\subsection{Konsequenzen für den Eliminierungstest}

Auf der Grundlage des Modells der Situationsvalenz kann auch erklärt werden, warum der Eliminierungstest, ein prinzipiell bewährtes strukturalistisches Verfahren zur Ermittlung von obligatorischen Konstituenten, bei der o/f-Klassifikation von Valenzstellen Probleme macht.

Beim Eliminierungstest wird aus einem Testsatz eine Valenzstelle entfernt. Wird der verbleibende Restsatz nicht mehr als grammatisch beurteilt, dann ist die Valenzstelle obligatorisch, ansonsten ist sie fakultativ. Der Eliminierungstest wird durchgeführt an selbstkonstruierten Testsätzen ohne Einbettung in einen Situations- und Redekontext. Dadurch fehlen aber genau diejenigen situations- und wissensbezogenen Parameter, die für die Realisierung von Situationsrollen - und darüber vermittelt über die Realisierung der entsprechenden Valenzstellen - von entscheidender Bedeutung sind.

Die in Abschnitt 2 angesprochene Tatsache, daß verschiedene Wörterbücher trotz Anwendung derselben Methode, nämlich des Eliminierungstests, zu unterschiedlichen o/fKlassifikationen für dieselbe Valenzstelle gelangen, kann nun folgendermaßen erklärt wer-

12 Die Beispiele stammen teilweise aus Arbeitsgruppe Marburg (1973) und Helbig (1992); weitere Beispiele in Storrer (1992: 298ff.). 
den: Eine Valenzstelle kann einmal als weglaßbar, ein andermal als nicht weglaßbar erscheinen, wenn der Beurteilende der "Restsätze” die Werte der valenzrelevanten Parameter verschieden auffüllt. Da der Test im allgemeinen für jede Valenzstelle nur einmal durchgeführt wird, variiert das Klassifikationsergebnis in Abhängigkeit von syntaktischen und semantischen Faktoren eines Testsatzes, die der Testperson eine bestimmte Auffüllung der valenzrelevanten Parameter suggerieren und somit die verschiedenen Filter beeinflussen. $\mathrm{Zu}$ diesen Faktoren zählen:

- Der im Testsatz vorliegende sprachliche Minimalkontext kann bewirken, daß ein Situationstyp assoziiert wird, bei dem die Belegung der in Frage stehenden Situationsrolle standardmäßig festliegt. Die Rolle wird vom Gesetzeswissensfilter herausgefiltert und muß nicht verbalisiert werden.

(7) Poker-Pepe gibt.

(8) * Die Vortragende gibt.

In (7) wird durch die Füllung des Nominativkomplements eine Rekurssituation vom Typ Kartenspiel nahegelegt. In diesem Situationstyp sind die Situationsrollen des Empfängers und des Objekts von geben standardmäßig festgelegt und können in der Kommunikation mit Insidern weggelassen werden. ${ }^{13}$ Satz (8) hingegen wirkt unvollständig, da eine entsprechende standardmäßige Rollenbelegung nicht durch den im Satz vorgegebenen Minimalkontext assoziiert werden kann.

- Weiterhin gibt es semantische Faktoren, die die Beurteilung der Relevanz einer Situationsrolle für das aktuelle Redethema und damit die Beurteilung der Weglaßbarkeit der entprechenden Valenzstelle beeinflussen. Zu diesen Faktoren zählen: ${ }^{14}$

- Habitualität: Wenn das Hauptaugenmerk im Satz darauf gerichtet ist, daß die vom Verb bezeichneten Handlungen/Ereignisse gewohnheitsmäßig stattfinden, können andere, das Verb weiter spezifizierende Komplemente, irrelevant und damit weglaßbar erscheinen.

(9) Er befiehlt immer.

(10) * Er befiehlt.

Satz (9) erscheint deshalb akzeptabler als Satz (10), bei dem der Minimalkontext keine habituelle Interpretation nahelegt.

- Generizität: ein Satz wie (11) hat eine generische Interpretation, bei der bestimmte Situationsrollen (in (11) die Rolle des Befehlenden) in die im Satz ausgedrückte gesetzesartige Aussage involviert sind.

13 Es sollte hierbei festgehalten werden, daß auch im Situationstyp Kartenspiel die Bedeutung von geben mit der Paraphrase $X$ macht, daß Y $Z$ hat umschrieben werden kann, so daß man nicht von einer situationstypspezifischen Spezialbedeutung ausgehen kann.

14 Vgl. Jacobs (1990) und Blume (1993). 
(11) Generale befehlen.

(12) Generale befehlen Soldaten.

Die Belegung anderer Situationsrollen (z.B. Befehlsempfänger und Befehl) hingegen bleibt offen; diese können, wie der Vergleich von (11) und (12) zeigt, unter kommunikativen Gesichtspunkten auch nicht hinzugefügt werden, ohne daß der Geltungsbereich für die betreffende gesetzesartige Aussagen eingeschränkt wird. In (10) dagegen ist keine generische Interpretation möglich; der Satz erscheint unvollständig und kann nur dann als akzeptabel gelten, wenn man ihm eine habituelle Lesart unterlegt.

- Kontrast: In Kontexten, in denen zwei Handlungen/Ereignisse durch Kontrastierung der entsprechenden Verben einander gegenübergestellt werden, sind zusätzliche Rollen irrelevant und können deshalb weggelassen werden.

(13) Er gehorcht nicht, sondern er befiehlt.

Wenn Valenzstellen auch in solchen Kontrastierungskontexten realisiert sein müssen, dann spricht dies tatsächlich für eine starke Bindung der Valenzstelle an das betreffende Verb, die allen kommunikativ-pragmatischen Einflüssen trotzt. Aus diesem Grund wird in Storrer (1992: 318ff.) auch eine modifizierte Form des von Pasch (1977) vorgeschlagenen Kontrasttests zur Ermittlung von perspektivierungsfixierten Rollen als Alternative zum Eliminierungstest propagiert.

- Obgleich die zum Eliminierungstest herangezogenen Testsätze nicht in sprachliche oder situationelle Kontexte eingebunden sind, kann durch Faktoren wie Wortstellung, Wahl des definiten oder des indefiniten Artikels nahegelegt werden, daß die Belegung einer Valenzstelle bereits vorerwähnt wurde. Dies beeinflußt den Hörerwissensfilter und damit die Beurteilung der Akzeptabilität der Restsätze.

(14) Ein Unfall hat sich ereignet.

(15) * Der Unfall hat sich ereignet.

In (14) wird durch den indefiniten Artikel assoziiert, daß das Ereignissubjekt für den Hörer neue Information enthält. Der Satz erscheint vollständig. Die Verwendung des definiten Artikels in (15) hingegen legt nahe, daß dem Hörer dieser Informationsteil bereits bekannt ist; das semantisch sehr unspezifische Verb sich ereignen eignet sich ebenfalls nur bedingt zum alleinigen Träger neuer Information. Der Satz erscheint nun in Bezug auf seine Informationsstruktur unvollständig.

Unter Kenntnis der Auswirkung der Faktoren können zu den meisten der in den Wörterbüchern vorgenommenen Klassifikationen systematisch Gegenbeispiele konstruiert werden. All diese Faktoren beeinflußen außerdem die im Modell der Situationsvalenz angelegten Filter, indem sie der Testperson eine bestimmte Auffüllung der valenzrelevanten Parameter nahelegen. Insofern vermutet Blume (1993) m.E. zurecht, daß die bereits angesprochene Uneinheitlichkeit der o/f-Klassifikation in den verschiedenen Wörterbüchern 
daher rührt, daß die herkömmliche Verfahrensvorschrift für die Durchführung des Eliminierungstests solche Faktoren nicht berücksichtigt. Vielmehr können beliebige Sätze Gegenstand der Eliminierungsexperimente sein, auch solche, die aufgrund ihrer syntaktischen oder semantischen Merkmale generell eine Nicht-Realisierung von Valenzstellen begünstigen. Blume (1993) versucht, das Problem der inkonsistenten Testergebnisse dadurch zu entschärfen, daß die o.g. Faktoren durch restriktivere Anweisungen zur Durchführung des Tests unter Kontrolle gehalten werden. Ihr modifizierter Eliminierungstest berücksichtigt sehr viele mögliche, auch syntaktische Störfaktoren: die Weglassung von Konstituenten ist nur im Mittelfeld und nur unter Verwendung von finiten Verbformen in der 3. Person Plural erlaubt; weiterhin sind bestimmte Interpretationen (u.a. Habitualität, Generizität und Kontrast) explizit auszuschließen.

Es muß jedoch im Auge behalten werden, daß mit der Einschränkung der Konstruktionen und Interpretationen, die beim Eliminierungstest verwendet werden dürfen, auch eine beträchtliche Einschränkung des Geltungsbereichs der o/f-Realisierungsregel einhergeht: Beanspruchte diese bislang Gültigkeit für alle Sätze mit dem in Frage stehenden Verb, so reduziert sich der Geltungsbereich nun auf die Sätze, in denen das betreffende Verb in der betreffenden Konstruktion vorkommt. Die entstehenden Klassifikationsergebnisse sagen deshalb letztlich wenig darüber aus, ob und wann Valenzstellen in Äußerungen, in denen die valenzrelevanten Parameter nicht kontrolliert werden, sondern frei variieren, verbalisiert oder nicht verbalisiert werden müssen. Eine derartige Reduktion des Geltungsbereichs ist nur dann unproblematisch, wenn der Einfluß der kontrollierten Faktoren auf die Weglassung der Valenzstellen systematisch und unabhängig vom Einzelverb und den übrigen valenzrelevanten Parametern (Situationstyp, Interessenlage, aktuelles Redethema etc.) ist. Dies muß für die einzelnen Faktoren empirisch überprüft werden.

\section{Zusammenfassung}

Ausgehend von den Problemen, die mit der dichotomischen Unterscheidung in obligatorische und fakultative Valenzstellen verbunden sind, wurde ein Modell zur Diskussion gestellt, das diejenigen Parameter in ihrem Zusammenspiel beschreibt, die die Wahl der in einer Äußerung zu realisierenden Verb-Rollen-Konstellation entscheidend beeinflussen. Dem Modell liegt die Annahme zugrunde, daß die Entscheidung, welche und wieviele Valenzstellen in einer Äußerung zu realisieren sind, in erster Linie durch kommunikativpragmatische Faktoren gesteuert wird; syntaktische und semantische Merkmale kommen erst in zweiter Linie zum Tragen. Entsprechend unterscheidet das Modell zwei hintereinandergeschaltete Schritte: Im einzelsprachunabhängigen Konzeptualisierungsschritt werden anhand von Parametern, die an die aktuelle Äußerungssituation gebunden sind, diejenigen konzeptuellen Rollen herausgefiltert, die die kommunikative Angemessenheit der geplanten Äußerung gewährleisten. Im einzelsprachspezifischen Verbalisierungsschritt wird anhand von syntaktischen und semantischen Eigenschaften entschieden, mit welchen Verben das konzeptuell gewählte Rolleninventar versprachlicht werden kann. Im Gegensatz zur o/f-Klassifikation beantwortet das Modell die Frage nach der Besetzung der Valenz- 
stellen also nicht nur unter dem Gesichtspunkt der grammatischen Korrektheit, sondern zusätzlich unter dem der kommunikativen Angemessenheit.

Das Modell verdeutlicht, daß die meisten Faktoren, die über die Realisierung von Valenzstellen in einer kommunikativ angemessenen Äußerung entscheiden, in Abhängigkeit vom Typ der Äußerungs- und der Rekurssituation variieren. Daraus ergeben sich einige interessante Konsequenzen für die lexikographische und grammatikographische Beschreibung der entsprechenden Regularitäten. So wurde gezeigt, daß die Unterscheidung von definiter und indefiniter Auslassung von Valenzstellen nicht auf der Ebene des Lexikons als feinere Subklassifizierung fakultativer Valenzstellen angelegt werden kann, da die Auslassung derselben Stelle, je nach Auffüllung der situationsspezifischen Parameter, definit oder indefinit zu interpretieren ist. Die durch empirische Untersuchungen bestätigten Probleme mit dem Eliminierungstest (dem methodischen Verfahren zur o/f-Klassifikation) wurden auf dem Hintergrund des Modells dadurch erklärt, daß die Eliminierung von Valenzstellen an Testsätzen ohne Einbettung in einen Äußerungskontext vorgenommen wird. Zur Beurteilung der reduzierten Sätze fehlen deshalb genau diejenigen situationsspezifischen Parameter, die für die Realisierung von Situationsrollen auf der konzeptuellen Ebene - und darüber vermittelt für die Verbalisierung der entsprechenden Valenzstellen entscheidend sind. Die Beurteilung des reduzierten Satzes wird deshalb wesentlich davon abhängen, wie der Beurteilende diese Parameter für sich auffüllt. Es wurden syntaktische und semantische Merkmale der zum Test herangezogenen Sätze diskutiert, die eine bestimmte Auffüllung dieser Parameter nahelegen und damit die Testergebnisse beeinflussen können.

\section{Literatur}

Arbeitsgruppe Marburg (1973): Aspekte der Valenztheorie. - In: Deutsche Sprache 1, 49-63.

Blume, Kerstin (1993): Valenz deutscher Verben und (Nicht-)Notwendigkeit. Arbeiten des SFB 282 Theorie des Lexikons, Nr. 48, Bergische Universität Gesamthochschule Wuppertal.

BW = Brockhaus-Wahrig: Deutsches Wörterbuch in sechs Bänden. Stuttgart, Wiesbaden 1981-1984.

Fillmore, Charles (1971): Types of Lexical Information. - In: Steinberg/Jacobovitz (Hg): Semantics. Cambridge, 370-392.

Gerzemysch-Arbogast, Heidrun (1987): Zur Thema-Rhema-Gliederung in amerikanischen Wirtschaftsfachtexten. Tübingen.

Grice, H.P. (1975): Logic and Conversation. - In: Cole/Morgan (Hg): Syntax \& Semantics 3: Speech Acts. New York, 41-58.

Helbig, Gerhard (Hg.) (1988): Valenz, semantische Kasus und/oder Szenen. Berlin (= Linguistische Studien, Reihe A, 180).

Helbig, Gerhard (1992): Probleme der Valenz- und Kasustheorie. Tübingen (= Konzepte der Sprach- und Literaturwissenschaft, 51).

HS = Helbig, Gerhard/Schenkel, Wolfgang (1991): Wörterbuch zur Valenz und Distribution deutscher Verben. 8. durchges. Auflage, Tübingen.

Heringer, Hans Jürgen (1984): Neues von der Verbszene. - In: Stickel, Gerhard (Hg.): Pragmatik in der Grammatik. Jahrbuch 1983 des Instituts für deutsche Sprache. Düsseldorf, 34-64.

Jacobs, Joachim (1990): Projektantrag zum Teilprojekt B2: Syntaktische Valenz im Lexikon. - In: Antrag zum SFB 282 Theorie des Lexikons, Heinrich-Heine-Universität Düsseldorf und Bergische Universität Gesamthochschule Wuppertal.

Mudersbach, Klaus (1981): Ein neues Thema zum Thema: Thema - Rhema. (Typoskript), Sprachwissenschaftliches Seminar der Universität Heidelberg. 
- (1984): Kommunikation über Glaubensinhalte. (= Grundlagen der Kommunikation und Kognition). Berlin/New York.

Pasch, Renate (1977): Zum Status der Valenz. - In: Linguistische Studien, Reihe A, 42, 1-50. Berlin

v. Polenz, Peter (1985): Deutsche Satzsemantik. Berlin/New York. (= Sammlung Göschen, 2226).

Sæbø, Kjell (1984): Über fakultative Valenz. - In: Deutsche Sprache 12, 97-109.

ViF = Schumacher, Helmut (Hg.) (1986): Verben in Feldern - Valenzwörterbuch zur Syntax und Semantik deutscher Verben. Berlin/New York. (= Schriften des IDS, 1).

Storrer, Angelika (1992): Verbvalenz. Theoretische und methodische Grundlagen ihrer Beschreibung in Grammatikographie und Lexikographie. Tübingen (= Reihe Germanistische Linguistik, 126).

Tesnière, Lucien (1959): Éléments de syntaxe structurale. Paris. 